\title{
The Reliability of Immunochromatographic Test in Tuberculous Children Diagnosed by Scoring System at Mohamed Elamin Hamid Hospital - Sudan
}

\author{
Duria Abdelraheim Mohammed Ahmed Omer ${ }^{1}$; Widad Alsheikh ${ }^{2}$; \\ Ahmed Yousif Adam ${ }^{3}$ \\ ${ }^{1}$ Associate prof. of Pediatrics-Omdurman Islamic University \\ ${ }^{2}$ Associate prof. of Paediatrics - Bahri University \\ ${ }^{3}$ MBBS Al Fashir University
}

\begin{abstract}
:
Background: Diagnosis of childhood tuberculosis (TB) is a challenging work for paediatrician. As many invasive procedures have been used with low yield. There is need for simple, rapid and feasible diagnostic test in diagnosis of childhood TB.

Aim of the study : To assess the reliability of immuochromotographic test (ICT) in diagnosis of childhood TB uses scoring system as gold standard.

Materials and Methods: Descriptive cross-sectional hospital-based study in the period from $1^{\text {St }}$ of April 2014 to $30^{\text {th }}$ of September 2014, conducted among 101child in Mohamed Elamin Hamid hospital. Informed consent was obtained from the parents. Firstly, all children were evaluated by scoring system for diagnosing TB. Then blood samples collected to perform ICT test for diagnosing TB. Diagnosed cases for TB by scoring system were tested for HIV, erythrocyte sedimentation rate (ESR) and haemoglobin level. In addition to chest X-ray. Weight and height were done for all children. Data were analyzed by using Statistical Package for Social Science (SPSS) then presented as tables and figures. Scoring system for diagnosing TB used as gold standard.

Results: out of 101 children there were 70 diagnosed as TB cases according to scoring system, 3 of them tested positive for ICT-TB. While, only 1 child test negative in the scoring system and test positive by ICT-TB. Sensitivity and specificity for ICT-TB were $4.3 \%$ and $96.8 \%$ respectively.

Conclusion: the study revealed low sensitivity of ICT-TB compared to scoring system.

Recommendation: ICT-TB could not be used in this setting and scoring system for diagnosing TB is highly recommended
\end{abstract}

\section{Introduction}

Tuberculosis (TB) is an infection caused by mycobacterium tuberculosis, responsible for high morbidity and mortality worldwide. It is one of the major causes of morbidity and mortality in Sudan although it is a curable and preventable disease, continues to be a leading cause of mortality and morbidity worldwide with an estimated 9 million new cases and 2 million deaths every year ${ }^{[1]}$. Early treatment of infectious cases reduces spreads of TB. Therefore, rapid and accurate identification of infected patients will help in good management ${ }^{[1,2]}$. Tuberculosis typically attacks the lungs, but can also affect other parts of the body. It spread through the air when people who have an active TB infection cough, sneeze, or otherwise respiratory fluids infected ${ }^{[2]}$. Most infections do not have symptoms, known as latent tuberculosis.

Tuberculosis (TB) is a contagious and airborne disease of poverty that mostly affects young adults in their most productive years in developing countries. WHO reported that one third of the world population is infected with latent TB with 9.4 million new overt cases annually ${ }^{[3]}$. Five to $10 \%$ of individuals with latent TB progress to develop overt disease during their life time ${ }^{[4-5]}$

Formal policies and control efforts addressing tuberculosis (TB) in children have been limited, in part due to lack of a standardized case definition and difficulties associated with establishing a definitive diagnosis ${ }^{[6]}$. However, diagnostic and treatment tools for TB in children have begun to be improving significantly. TB in children is now receiving more attention by researchers, clinicians, and policy makers. Estimating the global burden of tuberculosis (TB) in children is challenging due to the lack of a standard case definition, the difficulty in establishing a definitive diagnosis, the frequency of extra pulmonary disease in young children, and the relatively low public health priority given to TB in children related to adults ${ }^{[7]}$. The World Health Organization's (WHO) global TB data include age breakdowns only for smear-positive TB cases, among children, such cases are according to a small subset of the burden of disease due to TB (about 8 percent) ${ }^{[8]}$. The WHO estimates that of the 8.7 million incident cases of TB in 2011, approximately 500,000 occurred among children under age 15, also there were 64,000 pediatric deaths due to TB (among HIV-negative children) ${ }^{[9]}$. 
The Reliability of Immunochromatographic Test in Tuberculous Children Diagnosed by Scoring ..

Approximately $75 \%$ of these cases occurred in the 22 highest TB-burden countries ${ }^{[10]}$. As in many developing countries, children comprise more than one-half of the population, this makes the reported cases of childhood TB are likely underestimated.

Children under five represent an important demographic group for understanding TB epidemiology, since TB frequently progresses rapidly from latent infection to disease and severe disease manifestations, since miliary TB and meningitis, are more common in this age group, these children serve as sentinel cases, in recent and/or ongoing transmission of the disease in the community. Most children are infected by household contacts with TB cases, particularly parents or other caretakers. Even in circumstances when adult index cases are sputum smear-negative, transmission to children has been documented in 30 to 40 percent of households ${ }^{[11]}$.

Aim of the study: To assess the reliability of immuochromotographic test (ICT) in diagnosis of childhood TB using scoring system as gold standard.

\section{Study Design:}

\section{Material and Methods}

Descriptive observational cross-sectional hospital based study.

\subsection{Study Area:}

The study was conducted in Mohammed Elamin Hammed Hospital which is located in the centre of Omdurman Town beside Masjid Al-Khalifa, this hospital was donated by El Said Alamin Hammed in 1986. At that time, it consisted of 6 wards and 90 beds. The reconstruction and extension of the hospital was done in 2006. Now the hospital is composed of 10 wards (general and special) besides the emergency and outpatient department which consists of 24-hour admission wards, intensive care units (PICU and NICU), X-Ray department, laboratory, blood bank, pharmacy, facilities for doing ultrasound. The total number of beds now is 298.

\subsection{Study Period:}

$1^{\text {st }}$ April 2014 to $30^{\text {th }}$ Sept 2014.

2.3. Study Population:

All children who are suspected to have TB by scoring system and attending the outpatient in Mohammed Elamin Hammed Hospital.

\subsection{Inclusion Criteria:}

All children presented to outpatient at Mohammed Elamin Hammed Hospital are suspected to have TB and are diagnosed by scoring system.

Control was choosing from children that have not fulfilled the criteria of TB by the scoring system.

\subsection{Sampling Technique:}

Design: total coverage of patient suspected to have tuberculosis and attending the outpatient in Mohammed Elamin Hammed Hospital at the study period.

\section{Data Collection Tools:}

Questionnaire was composed of personal data, signs, symptoms, investigation (ICT, CXR, Mantoux, ESR, Sputum, weight and Height) and scoring system.

\subsection{Study Team:}

Author, House-officer, sister, and paediatric registrar work at hospital and they were oriented about the purpose of the study and method of data collection.

\subsection{Principal and Brand of the test:}

$5 \mathrm{ml}$ of blood collected in plain tube from every participant and allowed to clot, and then serum was tested for ICT for TB by follow the steps below:

1-Remove the test device from the foil pouch, place it on a flat dry surface.

2-Add $100 \mathrm{ml}$ of serum into the sample well (S) with micropipette.

3-As the test begins to work, you will see purple colour moving across the result window in the centre of the test device.

4- Interpret the test result within 15 minutes after sample application. Colour band will appear on the left section of the result window to show that the test is working properly; this band is the control band.

5- The right section of the result window indicates the test result, if another colour band appears on the right section of the result window, this band is the test band. 


\section{Results}

The current study was conducted among 101 children attended to outpatient at Mohammed Elamin Hammed Hospital as suspected TB. Out of 101 enrolled children, 70 were newly diagnosed as having tuberculosis by the scoring system and the rest were not fulfilling the criteria. The aim of the study was to compare the value of ICT test versus scoring system in diagnosis of tuberculosis.

Table (1): Gender and age group shows that, the majority of the study population found a common age group from 6 months to 5 years distributed as follows, $46(65.7 \%)$ have TB and $17(54.8 \%)$ without TB. Followed by 5-10 years' age group, $20(28.6 \%)$ patient have TB and $9(29 \%)$ without TB. Only 4(5.7\%) of those with TB, and $2(6.5 \%)$ of those without TB in more than 10 years. There are $3(9.7 \%)$ patients less than 6 months without TB.

Regarding gender distribution, the study shows that males were predominant $65(64.4 \%)$, in which $45(64.3 \%)$ of them have TB. Females were 36 (35.6\%), of whom 25 (35.7\%) have TB and $11(35.5 \%)$ without TB.

Figure (1): Shows the distribution of children among study population regarding the residence, the study revealed that, $76(75.2 \%)$ of study population are from Omdurman $61(87.1 \%)$ with TB and $15(48.4 \%)$ without it, $17(16.8 \%)$ from Khartoum $7(10 \%)$ with TB, and $10(32.2 \%)$ have not. $2(6.5 \%)$ patients from Bahri without TB, $6(6 \%)$ children from outside Khartoum State 2 of them $(2.9 \%)$ with TB and $4(12.9 \%)$ without it.

Figure (2): Regarding symptoms and signs among study population, chronic cough found to be common symptom which was seen in $63(90 \%)$ in those with TB and $14(45.2 \%)$ in those without TB, followed by weight loss in $52(74.3 \%)$ in TB, and 5(16.2\%) in children without TB. prolong fever in 47(67.2\%) with TB and 10 $(32.3 \%)$ in those without TB, Lymphadenopathy in 13(18.6\%) and night sweating in $7(10 \%)$ of children, clubbing finger in $3(4.3 \%)$. Only $2(2.9 \%)$ of children with tuberculosis were found to have abdominal lymph nodes. No one of children with suspected TB have Lymphadenopathy, night sweating or clubbing.

Figure (3): The majority of study population $65(64.4 \%)$, were found to scoring system > 5, 5(5\%) of them have score of 5 , and $31(30.7 \%)$ score less than 5.

Table (2): According to BCG vaccine, 90 (89.1\%) of study population were received vaccine. Those with TB, $59(84.3 \%)$ were vaccinated and 11(15.7\%) were not. All children without TB $31(100.0 \%)$ are vaccinated and have BCG scar.

Table (3): ICT versus scoring system show that out of 101 cases suspected having TB there is 70 previously diagnosed positives by scoring system, ICT identified 3 of them as positive (true positive) and 67 as negative (false negative). Out of 31 participants, ICT identified one case not fulfil the criteria as positive (False positive) and 30 as negative (true negative).

Table (4): There was history of contact among 39 (55.7\%) with patient of TB, and 31(44.3\%) not.

Table (5) and Figure (4): Regarding the investigation result, most of children with TB 67(95.7\%) found to have negative smear sputum for ZN stain for Acid Fast Bacilli, and only 3(4.3\%) have positive to ICT. One child showed positive result of HIV test. Mantoux test found positive in 57(81.4\%) of children, while 13(18.6\%) of children showed negative result. Among positive results, 53(75,7\%) recorded scar of 5-10 mm, $10(14.3 \%)$ recorded scar of $<5 \mathrm{~mm}$ and $7(10 \%)$ recorded scar of more than $10 \mathrm{~mm}$.

Figure (5): ESR of $50-100 \mathrm{~mm} / \mathrm{hr}$ was read in 28(40\%) of children, less than 50 were read in 26(37.1\%) and more than 100 were read in $16(22.9 \%)$.

Figure (6): Chest X-ray findings found normal in 44(62.9\%), consolidation was more common in patient with TB $24(34.3 \%)$, and pleural effusion in two children $(2.9 \%)$.

Table (6): Nutritional status among study population show that $51(50.5 \%)$ of children well-nourished and have TB and 31(30.7\%) without it, 19(18.8\%) were malnourished, among which 8 children were (< - 1 SD), 6 children were $(<-2 \mathrm{SD})$ and 5 children were $(<2-3 \mathrm{SD})$.

Table 1: Gender and age group distribution among study population $(n=101)$

\begin{tabular}{|c|c|c|c|}
\hline Variables & $\begin{array}{c}\mathbf{T B} \\
\mathbf{N}=\mathbf{7 0}\end{array}$ & $\begin{array}{c}\text { Without TB } \\
\quad \mathrm{N}=31\end{array}$ & $P$-value \\
\hline $\begin{array}{l}\text { Gender } \\
\text { Male }\end{array}$ & $45(64.3 \%)$ & $20(64.5 \%)$ & \multirow{2}{*}{$\begin{array}{l}0.642 \\
\text { NS }\end{array}$} \\
\hline Female & $25(35.7 \%)$ & $11(35.5 \%)$ & \\
\hline $\begin{array}{l}\text { Age groups } \\
\text { Less than } 6 \text { months }\end{array}$ & $0(0.00 \%)$ & $3(9.7 \%)$ & \multirow{4}{*}{$\begin{array}{l}0.281 \\
\text { NS }\end{array}$} \\
\hline 6 months -5 years & $46(65.7 \%)$ & $17(54.8 \%)$ & \\
\hline $5-10$ years & $20(28.6 \%)$ & $9(29 \%)$ & \\
\hline More than 10 years & $4(5.7 \%)$ & $2(6.5 \%)$ & \\
\hline
\end{tabular}

$\mathrm{NS}=$ not statistically significance 
The Reliability of Immunochromatographic Test in Tuberculous Children Diagnosed by Scoring ..

Table (2): BCG Vaccine among study population $(\mathrm{n}=101)$

\begin{tabular}{|c|c|c|c|c|}
\hline Variable & \multicolumn{4}{|c|}{ Total population } \\
\hline \multirow{3}{*}{ Status of BCG vaccine } & & $\begin{array}{c}\text { TB } \\
\mathrm{N}=70\end{array}$ & $\begin{array}{c}\text { Without TB } \\
N=31\end{array}$ & $P$-value \\
\hline & Yes & $59(84.3 \%)$ & $31(100 \%)$ & \multirow{2}{*}{$\begin{array}{l}0.316 \\
\text { NS }\end{array}$} \\
\hline & No & $11(15.7 \%)$ & $0(0.0 \%)$ & \\
\hline
\end{tabular}

$\mathrm{NS}=$ not statistically significance

Table 3: ICT versus scoring system in diagnosis of TB $(n=101)$

\begin{tabular}{|l|l|c|c|c|}
\hline \multirow{2}{*}{ ICT for TB } & \multicolumn{2}{|c|}{ Scoring System } & \\
\cline { 2 - 5 } & & Positive & Negative & Total \\
\cline { 2 - 5 } & Test Positive & 3 & 1 & 4 \\
\cline { 2 - 5 } & Test Negative & 67 & 30 & 97 \\
\hline Total & 70 & 31 & 101 \\
\hline
\end{tabular}

Table 4: History of contact in children with TB $(n=70)$

\begin{tabular}{|c|c|c|}
\hline History of contact & Frequency & \% \\
\hline Yes & 39 & 55.7 \\
\hline No & 31 & 44.3 \\
\hline Total & 70 & 100.0 \\
\hline
\end{tabular}

Table 5: Laboratory Investigations for TB patient $(n=70)$

\begin{tabular}{|l|c|c|}
\hline \multirow{2}{*}{ Investigations } & \multicolumn{2}{|c|}{ Investigation } \\
\cline { 2 - 3 } & \multicolumn{1}{|c|}{ Test Positive } & $67(95.7 \%)$ \\
\hline Smear stain ZN stain & $3(4.3 \%)$ & $97(96 \%)$ \\
\hline ICT & $4(3.9 \%)$ & $13(18.6 \%)$ \\
\hline Mantoux test & $57(81.4 \%)$ & $69(98.6 \%)$ \\
\hline HIV test & $1(1.4 \%)$ & \\
\hline
\end{tabular}

Table 6: Nutritional status among study population $(n=101)$

\begin{tabular}{|l|l|l|l|c|}
\hline \multicolumn{2}{|l|}{} & \multicolumn{1}{|c|}{$\begin{array}{c}\text { Children with TB } \\
\mathbf{N = 7 0}\end{array}$} & $\begin{array}{c}\text { Children without TB } \\
\mathbf{N}=\mathbf{3 1}\end{array}$ & \multirow{2}{*}{$\boldsymbol{P}$-value } \\
\hline & Normal weight & $51(72.9 \%)$ & $31(100.0 \%)$ & \\
\hline & $<-3 S D$ & $19(27.2 \%)$ & $0(0 \%)$ & $<0.001 *$ \\
\hline
\end{tabular}

*Statistical significantly differences

\section{Discussion}

Rapid and accurate diagnosis of tuberculosis (TB) is crucial to facilitate early treatment of infected cases, in order to reduce its spread. The diagnosis of childhood TB is a difficult job, and occasionally need invasive procedure like sputum collection and gastric aspiration which are too harm for children. To improve the diagnosis of TB in children, more simple and rapid diagnostic test is needed. Therefore, the current study attempted to assess immunochromatographic (ICT) test as a new diagnostic test which is simple, rapid, available and not invasive to detect active cases in children to enable clinician to start early treatment. In this study, sensitivity and specificity of ICT test for tuberculosis was assessed for the first time in Sudan.

The current study was conducted to assess ICT reliability and determine its sensitivity and specificity in diagnosis of TB compared to Scoring system. One hundred one children were suspected to have TB, seventy children were diagnosed to have TB by positive scoring system method and 31 not fulfil the criteria. The result showed that, out of 70 children with TB, only 3 children $(4.3 \%)$ showed positive result in ICT test considered the sensitivity (4.3\%) and showed high specificity (96.8\%); out of 31 children suspected TB, only one (3.2\%) was found to have positive ICT. identified 30 children as negative (true negative) and one child as positive (false positive). This result compatible with the study done in (Algeria) which revealed poor sensitivity and high specificity of ICT test; the sensitivities of ICT was $(2.3 \%)$, while its specificity was $100 \%{ }^{[12]}$. There was study done in Sudan by Ali compatible with our result which was very low about $2 \%$ the sensitivity. ICT test in the current study didn't provide a promising result of detecting positive cases of tuberculosis. Recently, Gozde Ongut and colleagues reported that, ICT test has low sensitivity $33.3 \%$, and high specificity $100 \%$, these findings not far from our result. ${ }^{(13)}$ In contrast, other study in Korea reported the sensitivity and specificities of ICT were 73 and $88 \%$ respectively ${ }^{[14]}$.

Regarding age distribution, most of children with TB, found at age group of 6 months to 5 years, followed by $5-10$ years which represented by $29.5 \%$ and $6.8 \%$ of children more than 10 years. Literature has reported that, younger children are more vulnerable to rapid progression of TB infection and developing its severity such as miliary TB and meningitis, are more common in this age group. Therefore, these children serve 
as sentinel cases, indicating recent and/or ongoing transmission in the community. ${ }^{[15]}$ According to gender, the study revealed that, male predominated to female, with male to female ratio of 1.8: 1 . Shakak and colleagues showed an incompatible resulting reporting that, male: female ratio was 1:4. ${ }^{[10]}$

The most frequent symptoms of tuberculosis reported were chronic cough (90\%), weight loss $(74.3 \%)$, prolong fever $(67.2 \%)$ and Lymphadenopathy was reported in less cases. This is compatible with previous reported by the $\mathrm{WHO}^{[10]}$ A persistent, non-remitting cough and fever were exclusively reported in children with tuberculosis in the study conducted by Marais, et al. in 2005, they concluded that, the use of well-defined symptoms is feasible, even in resource limited settings, and may offer significantly improved value in the diagnosis of childhood pulmonary tuberculosis. ${ }^{[15]}$.

A considerable number of children with TB in the study found to have contact with TB patients (55.7\%). This is compatible with previous literature which revealed that, most children are infected by household contacts with TB disease, particularly parents or other caretakers. ${ }^{[15]}$ Refugees, migration and low health education always create suitable environment to spread the infection through contact.

Vast majority of children reported score more than $5(64.4 \%)$, while few $(5 \%)$ had score of five. Among the 70 cases identified positive by scoring system, this is similar to findings reported in Ministry of Health in Brazilian, they used Keith Edward scoring system (authorized by the WHO to diagnose TB). They revealed good sensitivity to diagnose TB (possible tuberculosis), with $92 \%$ sensitivity, thus feasible for use as an ancillary diagnostic test in children ${ }^{[16]}$.

Regarding BCG vaccine, the study revealed that (84.3\%) of children with TB found to be vaccinated, while only $15.7 \%$ were not vaccinated. A study done by Richard and college found that TB is $4 \%$ among vaccinated and $11 \%$ among non-vaccinated. BCG vaccination reduced the risk of TB, yielding a vaccine effectiveness of $50 \% .^{(17)}$ Although there are great efforts to decrease rate of tuberculosis in Sudan, still their difficulty of controlling the outbreak of the disease in certain areas. Tuberculin skin test (Mantoux test) showed predomination of $5-10 \mathrm{~mm}(75.7 \%)$, and few percentages were $<5 \mathrm{~mm}(14.3 \%)$ and $>10 \mathrm{~mm}(10 \%)$. This is inconsistent with findings reported by Al Marri who reviewed that, out of 306 participants, tuberculin skin test was less than $5 \mathrm{~mm}$ in $(8.2 \%), 5-10 \mathrm{~mm}$ in $(1.6 \%)$, more than $10 \mathrm{~mm}$ in $(90.2 \%)^{[18]}$.

Most children with pulmonary TB in the current study showed normal result in X-ray examination, while more than third of them showed consolidation and very few $(2.9 \%)$ showed pleural effusion. The most prevalent abnormalities in the positive smear group were consolidation, tree-in-bud pattern, upper lobe nodular infiltration and cavitations ${ }^{[19]}$.

Regarding nutritional status of children with $\mathrm{TB}$, the study revealed that $27.2 \%$, found to be malnourished. There is strong relationship between malnutrition and TB, study done in Mallawi in 2000 found that $34 \%$ of the severely malnourished children admitted to the Blantyre Queen Elizabeth hospital nutritional rehabilitation unit were to have TB. ${ }^{(20)}$

\section{Conclusion}

$>$ According to the study findings, sensitivity and specificity of ICT-TB $4.3 \%$ and $96.8 \%$ compared with scoring system for diagnosing TB. This low sensitivity is not safe in diagnosing childhood TB. As many factors can affect the ICT result.

$>$ Given the scoring system, it is reliable based on clinical data that is easy to notice by clinician enable them rapidly diagnose the child and start the management immediately.

$>$ Therefore use of scoring system in this study is favoured over using ICT test as diagnostic for TB.

\section{Recommendations}

1. Diagnosis of childhood TB could be relying on scoring system alone as obtaining bacteriological diagnosis is extremely difficult.

2. The ICT test is not reliable in the diagnosis of tuberculosis; it may need further study with larger sample size to approve its validity.

3. BCG Vaccine should be done for all children.

4. Any child should be monitored for his nutritional status and mother should be counciled regarding their feeding.

5. Any child with malnutrition should be investigated for TB.

\section{References}

[1]. World health organisation; global tuberculosis control; WHO report 2010.Geneva; world health

[2]. Organization 2010[http;//www.who.int/tb/publications/global-report/2010/en/index.html] Adjei AA, Armah H, Duah OA, Adiku T, Hesse IFA. Evaluation of a rapid serological chromatographic immunoassay for the diagnosis of pulmonary tuberculosis in Accra, Ghana. Jpn J Infect Dis. 2003;56:161-164.

[3]. WHO Report. Global Tuberculosis Control. 2010. WHO/HTM/TB/2010.7. 
The Reliability of Immunochromatographic Test in Tuberculous Children Diagnosed by Scoring ..

[4]. Machado A, Emodi K, Takenami I, Finkmoore BC, Barbosa T, Carvalho J, Cavalcanti L, Santos G, Tavares M, Mota M, Barreto F, Reis MG, Arruda S, Riley LW. Analysis of discordance between the tuberculin skin test and the interferon-gamma release assay. IJTLD. 2009;13:446-453.

[5]. Shakak AO, Khalil EAG, et al. Possible risk factors of progression to overt disease among individuals with latent tuberculosis infection in the Sudan. IJCR. 2013;13:1107-1110.

[6]. Starke JR. New concepts in childhood tuberculosis. Curr Opin Pediatr 2007; 19:306.

[7]. Nelson LJ, Wells CD. Global epidemiology of childhood tuberculosis. Int J Tuberc Lung Dis 2004; 8:636.

[8]. Wells CD, Nelson LJ. New international efforts in childhood tuberculosis: proceedings from the 2002 Workshop on Childhood Tuberculosis, Montreal, Canada, 6-7 October 2002. Int J Tuberc Lung Dis 2004; 8:630.

[9]. World Health Organization. Global tuberculosis control: WHO report 2012. Geneva: World Health Organization, 2012. WHO/HTM/TB/2012.16.

[10]. Stop TB Partnership Childhood TB Subgroup World Health Organization. Guidance for National Tuberculosis Programmes on the management of tuberculosis in children. Chapter 1: introduction and diagnosis of tuberculosis in children. Int $\mathrm{J}$ Tuberc Lung Dis 2006; 10:1091.

[11]. Marais BJ, Gie RP, Schaaf HS, et al. The clinical epidemiology of childhood pulmonary tuberculosis: a critical review of literature from the pre-chemotherapy era. Int J Tuberc Lung Dis 2004; 8:278.

[12]. Walid Ben-Selma, Harizi H, Boukadida J. Immunochromatographic IgG/IgM Test for Rapid Diagnosis of Active Tuberculosis. Clin Vaccine Immunol. 2011 Dec; 18(12): 2090-2094.

[13]. Ongut G, Ogunc D, et al. Evaluation of the ICT Tuberculosis test for the routine diagnosis of tuberculosis. BMC Infect Dis. 2006; 6: 37.

[14]. Chang CL, Lee EY, Son HC, Park SK. Evaluating the usefulness of the ICT tuberculosis test kit for the diagnosis of tuberculosis. J Clin Pathol. 2000 Sep;53(9):715-7.

[15]. Marais BJ, Gie RP, Schaaf HS, et al. The clinical epidemiology of childhood pulmonary tuberculosis: a critical review of literature from the pre-chemotherapy era. Int J Tuberc Lung Dis 2004; 8:278.

[16]. Maciel EL, Dietze R, Silva RE, Hadad DJ, Struchiner CJ. [Evaluation of a scoring system recommended by the Brazilian Ministry of Health for the diagnosis of childhood tuberculosis]. Cad Saude Publica. 2008 Feb;24(2):402-8.

[17]. Richard. D . and college, BCG and infection with Mycobacterium tuberculosis, Published Online First: 3 October 2014 doi:10.1136/thoraxjnl-2014-206128

[18]. Al Marri M. The tuberculin skin test in confirmed pulmonary tuberculosis in the state of Qatar: where we stand?. Qatar Med J. 2012(2): 16-19.

[19]. Bolursaz MR, Mehrian P, et al. Evaluation of the relationship between smear positivity and high-resolution x.ray findings in children with pulmonary tuberculosis. Pol J Radiol. 2014 May 26;79:120-5.

[20]. Puoane T, Sanders D, Chopra M, et al. Evaluating the clinical management of severely malnourished children-a study of two rural district hospitals. S Afr Med J 2001; 91:137-41. 